\title{
Transcervical excision of intramasseteric cavernous hemangioma: A case report
}

\author{
YU-TING CHENG ${ }^{1}$ and CHIEN-CHUNG LAI ${ }^{1,2}$ \\ ${ }^{1}$ Department of Otolaryngology, Taipei City Hospital Renai Branch, Taipei City 106; \\ ${ }^{2}$ Department of Otorhinolaryngology, Faculty of Medicine, National Yang-Ming University, Taipei City 102, Taiwan R.O.C.
}

Received November 28, 2014; Accepted November 23, 2015

DOI: 10.3892/ol.2016.4105

\begin{abstract}
Intramuscular hemangiomas (IMHs) of the masseter muscle are extremely rare in the head and neck region and, thus, are often misdiagnosed as parotid tumors prior to surgery. Excisional resection remains the standard treatment for IMH. Since these tumors are located on the proximal side of the facial nerve, it is important to preserve the facial nerve during surgery. This study reports the case of a 57-year-old male who presented with a progressive tender swelling on the right side of the face, which had been present for $>6$ months. Computed tomography of the neck revealed a heterogeneous highly-vascularized mass located in the superficial layer of the masseter muscle. The patient subsequently underwent surgical resection via a collar incision, and pathological examination revealed a cavernous IMH. During the one-year follow-up period, the patient exhibited a good prognosis, and one-year magnetic resonance imaging revealed no local recurrence.
\end{abstract}

\section{Introduction}

Intramuscular hemangioma (IMH) of the head and neck region is extremely rare, accounting for $<1 \%$ of all cases of hemangioma in Caucasian populations (1). In America, IMHs accounted for $\sim 0.8 \%$ of all benign vascular neoplasms in 1940 (1), and in $197513.8 \%$ of IMHs were observed in the head and neck region (2). The masseter muscle is the most common region, followed by the trapezius and sternocleidomastoid muscle (3). IMHs predominantly occur before the age of 30 years without gender predominance (4); however, intramasseteric IMH is male-dominated (3). There are two major hypotheses for IMH: The first is the hereditary type and the second type is trauma-related (4). The presenting symptoms and signs include gradually enlarging lesions leading to cosmetic problems and certain IMHs are associated with

Correspondence to: Dr Chien-Chung Lai, Department of Otolaryngology, Taipei City Hospital Renai Branch, No. 10, Section 4, Ren'ai Road, Daan, Taipei City 106, Taiwan R.O.C. E-mail: DAL18@tpech.gov.tw

Key words: intramuscular hemangioma, masseter muscle, parotid tumor, parotidectomy, vascular tumor pain (3). Due to the location and characteristics of IMHs, it is difficult to make a correct diagnosis prior to surgery (5). IMH is often misdiagnosed as salivary neoplasms. Other differential diagnoses include cysts, lymphangiomas, rhabdomyosarcomas, masseteric hypertrophy and schwannomas (3). The treatment is variable, including cryotherapy, radiotherapy and steroid injection $(4,6)$; however, surgery is the primary treatment strategy $(3,7,8)$. When intramasseteric IMHs are located proximal to the facial nerve, an approach of superficial parotidectomy with facial nerve preservation and masseter muscle excision is generally adopted $(3,4,7,8)$. In consideration of local recurrence, total excision of the masseter muscle may be recommended (3). However, total extirpation of a suitable intramasseteric IMH with functional preservation of the facial nerve and masseter muscle may be achieved via a transcervical approach, which shortens the duration of the operation and hospital stay, and also significantly improves cosmesis. The current study presents the case of a 57-year-old male with intramasseteric IMH who underwent surgery via the less invasive route. In addition, available literature regarding the clinical radiology, pathology and treatment of intramasseteric IMH are reviewed. Written informed consent was obtained from the patient.

\section{Case report}

In July 2011, a 57-year-old male with no previous medical history presented to the outpatient department at Taipei City Hospital Renai Branch (Taipei City, Taiwan) with a painful mass on the right side of the face, which had been present for $\sim 6$ months. Physical examination identified a $3 \times 3 \mathrm{~cm}$, movable mass in the right cheek, located just above the mandible. The mass became firmer upon forceful mastication. Nasopharyngoscopy revealed a smooth nasopharynx and no evidence of tumor in the upper aerodigestive tract. Computed tomography (CT) of the neck showed a highly-vascularized, heterogeneous mass, $3 \mathrm{~cm}$ in diameter, in the superficial layer of the masseter muscle, with intratumor whirl-like contrast enhancement (Fig. 1). Fine needle aspiration identified no malignant cells, and the infiltration of a small number of eosinophils and lymphocytes was observed.

The patient was subsequently referred for tumor excision. Under general anesthesia, a collar incision of $\sim 5 \mathrm{~cm}$ was made in the submandibular region, approximately two-finger widths 
below the inferior border of the mandible. The platysmal flap was then elevated cephalad, and the mandibular branch of the facial nerve was identified and preserved. The tumor at the superficial layer of the masseter muscle was removed en bloc (Fig. 2). After hemostasis and placing of the drainage tube, wound layered closure was performed and the wound was dressed with dry gauze. The drainage tube was removed two days after surgery, and the right corner of the patient's mouth regained normal movement.

Based on pathological examination of the excised specimen, a diagnosis of cavernous IMH was determined (Fig. 3). No complications occurred during the postoperative period and no further treatment was administered. The functional and cosmetic results were excellent, and no recurrence was identified on the 1 year magnetic resonance imaging (MRI) follow-up scans (Fig. 4). To date, the 4-year follow-up course has remained uneventful.

\section{Discussion}

Hemangioma is the most common type of benign tumor in infants $(6,9)$, and also one of the most common manifestations of birth defects (9). Hemangioma in skeletal muscles accounts for only $\sim 0.8 \%$ of all benign vascular neoplasms (3), and $15 \%$ of IMHs occur in the head and neck region (6). The masseter muscle is the most common location, followed by the trapezius and sternocleidomastoid muscles (3). In the majority of cases, IMH occurs in patients $<30$ years of age, with no gender predilection $(3,5)$.

Among the classification methods of vascular tumors, the histological classification is most commonly accepted (4). According to Allen and Enzinger's definition of hemangiomas, these tumors are classified into a large vascular type (vessel diameter, $>140 \mathrm{~mm}$ ), small vascular type (vessel diameter, $<140 \mathrm{~mm}$ ) and mixed vascular type corresponding to cavernous, capillary and mixed types, respectively (10). This classification is useful and is consistent with clinical performance and recurrence (10). Capillary-type IMH is the most common, accounting for $50 \%$ of all skeletal muscle hemangiomas (4). It occurs most commonly in the head and neck region, accounting for $\sim 68 \%$ of cases $(4,8)$. Histologically, capillary-type IMH is hypercellular and thus, it is difficult to diagnose preoperatively (10). By contrast, the cavernous- and mixed-types of IMHs occur most commonly in the lower extremities and trunk, with reported incidence rates of 26 and $5 \%$, respectively $(8,11)$. These types of tumor tend to have prolonged symptoms and, thus, such tumors are typically diagnosed earlier than capillary-type IMH. Notably, mixed-type IMH exhibits the highest recurrence rate, at $\leq 28 \%(8,10,11)$.

IMHs gradually increase in size, typically over a period of $<1$ year (6). The most common manifestation is a painful lump, with $50-60 \%$ of patients reporting pain. However, pain is not the only symptom in patients with tumors $>3 \mathrm{~cm}$ in size (6). There are generally no overlying skin changes, and thrills, bruits, compressibility and pulsation are typically absent; however, situations that increase the venous pressure in the head increase IMH size (5). The 'turkey wattle' sign, a swelling that becomes most apparent when the head is in a dependent position, may be observed $(8,11)$. Forceful mastication can cause the lesion to
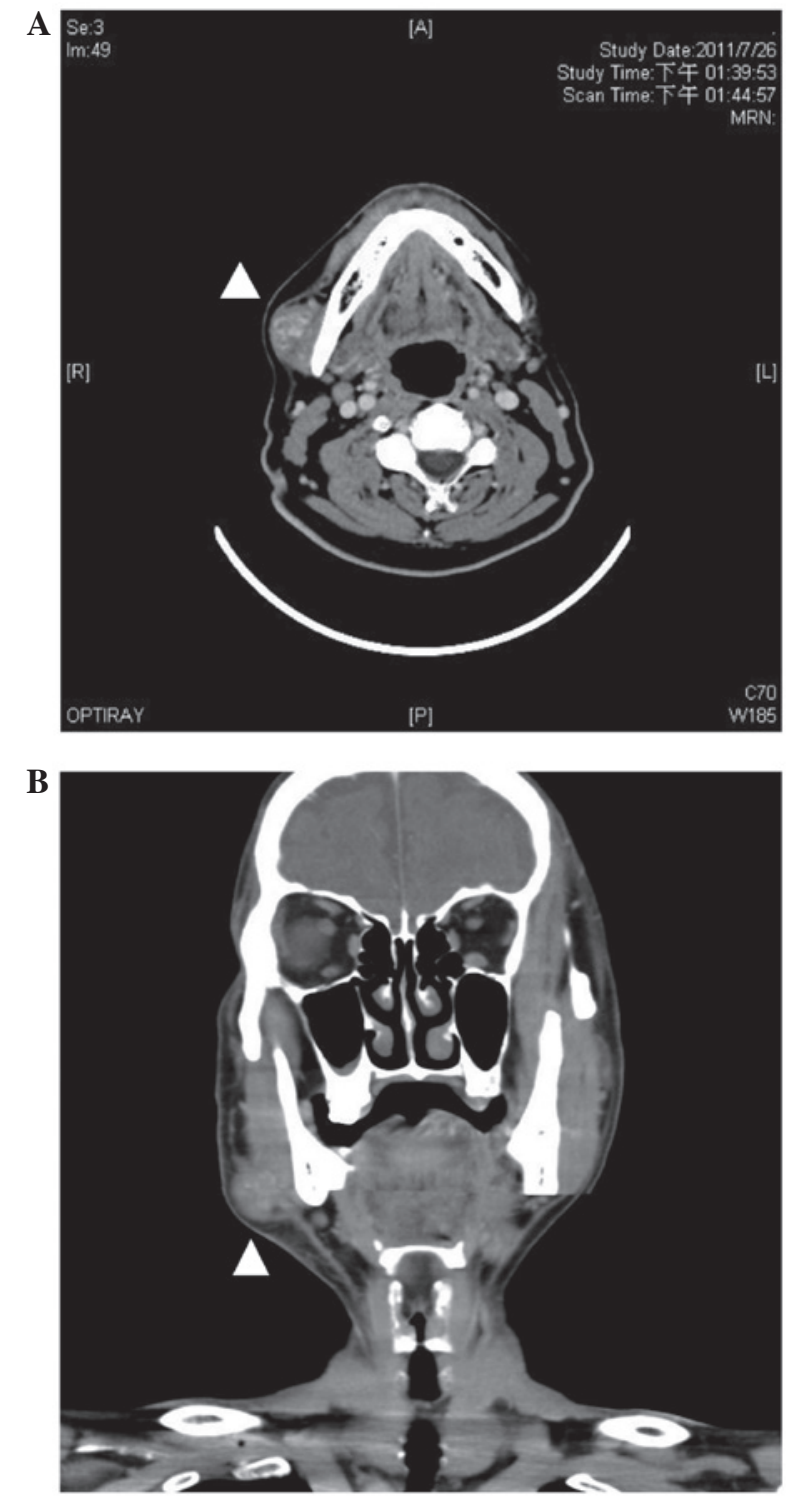

Figure 1. Computed tomography identified a tumor at the superficial layer of the right masseter muscle with whirl enhancement (white arrow head). (A) Axial and (B) coronal view.

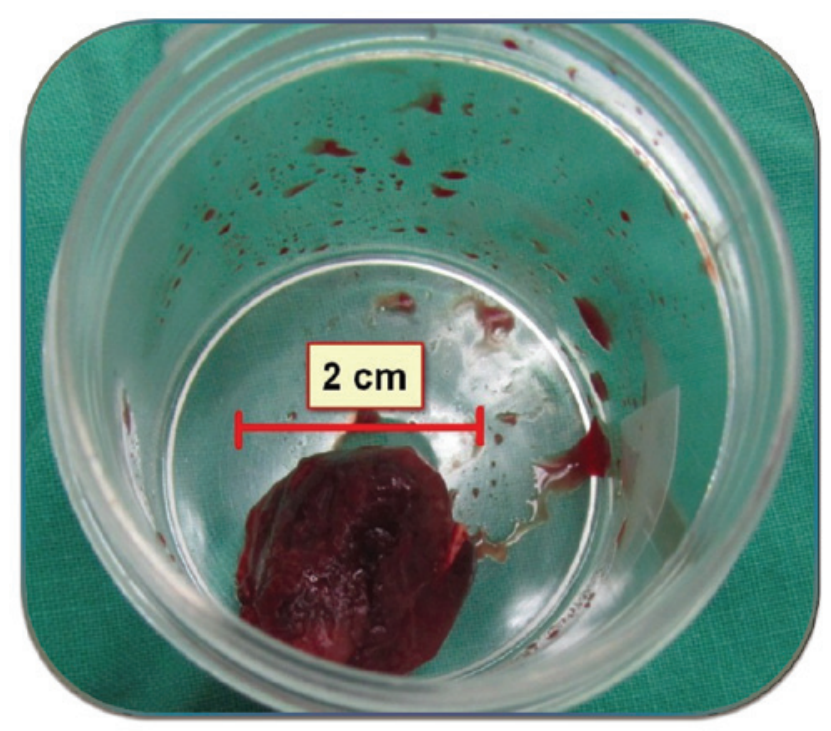

Figure 2. Gross tumor specimen removed via en bloc resection. 

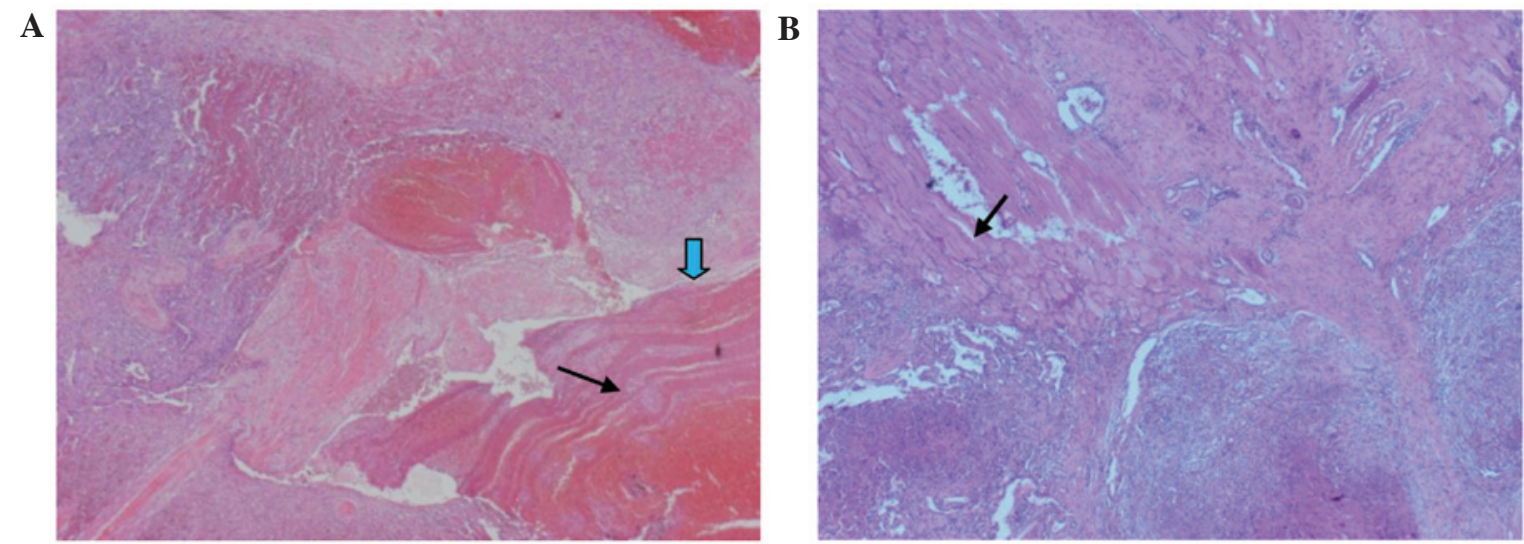

Figure 3. Pathological examination revealed a cavernous-type hemangioma. Venous-type vessels with a large lumen and thin wall infiltrated with striated muscle bundles were identified. In addition, thrombus organization in the lumen was observed. (A) Vascular spaces (blue arrow) filled with organizing thrombi (black arrow) (stain, hematoxylin and eosin; magnification, x40). (B) Cystically dilated vascular spaces extending between individual skeletal muscle fibers (thin black arrow) (stain, hematoxylin and eosin; magnification, $\mathrm{x} 400$ ).

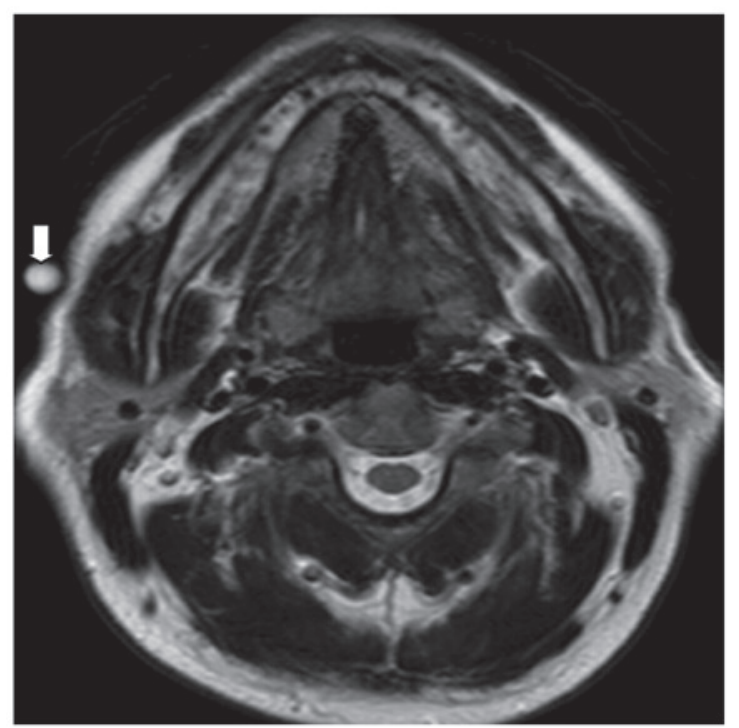

Figure 4. Magnetic resonance imaging examination performed 1 year following surgery identified no recurrence of the tumor, as marked by a capsule of cod liver oil (arrow).

become hard and difficult to move, as observed upon preoperative physical examination in the present case.

Correctly diagnosing IMH prior to surgery is extremely difficult; the tumor occurs within the muscle and overlaps the parotid gland; thus, $<8 \%$ of IMH cases are diagnosed preoperatively (10). Based on the location and morphology of the tumor, IMH may be differentiated from other benign and malignant tumors, including neurofibromatosis, aneurysms, lymphangiomas, rhabdomyosarcomas, masseteric hypertrophy, schwannomas and other head and neck malignancies (10). It is difficult to make a definitive diagnosis clinically by fine needle aspiration cytology, as pathology often only shows blood cells. In the present case, fine needle aspiration cytology ruled out malignancy; however, it did not provide any further information. Hemangioma was not suspected, therefore, arteriography was not arranged. Although arteriography and flow dynamics may confirm the existence of a hemangioma and its vascular type, the low-flow lesion or capillary-type of IMH makes the diagnosis more difficult. Furthermore, plain films have not been reported to be useful in the diagnosis $(5,7)$. With regard to CT and MRI, the majority of case reports indicate that MRI is of more diagnostic value for this type of soft tissue tumor than CT. However, in the present case, CT scans showed a $3.1-\mathrm{cm}$ slightly heterogeneous iso-dense lesion in the superficial portion of the right masseter muscle. Following injection of the contrast agent iopamidol (Iopamiro 370; Bracco, High Wycombe, UK) during the CT exam, heterogeneous whirl-like enhancement was noted rapidly, revealing a highly-vascularized lesion (Fig. 1). Therefore, hemangioma, arteriovenous malformation and vascular-rich malignancies were all considered. However, as the lesion exhibited an intact border and no invasion was evident, the possibility of malignancy was ruled out and IMH was diagnosed. Therefore, the results of the present case indicate that CT is helpful for the accurate diagnosis of IMH.

Appropriate treatment must be selected based on the conditions of the case; factors to be considered include patient age, tumor size, location and depth of invasion $(3,4)$. 
Various treatment modalities have been reported, including cryotherapy, radiation therapy, steroid injections, vascular thrombosis, injections of sclerosing material and surgical excision $(5,6)$. However, surgery remains the most common treatment for IMH. Determining the tumor entity during surgery is a major difficulty due to the tumor's ambiguous capsule. The local recurrence rate following surgical resection is 9-28\%; however, removing the masseter muscle completely has been reported to reduce the recurrence rate (6). In addition, the facial nerve may be damaged easily during surgery. Surgery to remove an IMH in the head and neck region may be approached transorally or via superficial parotidectomy with a preauricular incision $(6,12)$. Superficial parotidectomy with a preauricular incision removes the tumor completely and preserves the facial nerve with minimal damage, and thus it should be performed when tumors proximal to the parotid gland require resection. Previous studies have also reported that parotidectomy is a safer technique for the removal of rare tumors located in the head and neck region compared with a transoral approach. Additionally, this type of surgery reduces the risk of recurrence $(6,12,13)$.

In the present case, a collar incision of the neck was used instead of a preauricular incision. This approach was considered appropriate for the patient for the following reasons: Firstly, a smaller wound results in a shorter operative time, whilst allowing for complete removal of the tumor, as the tumor was small in size and located at an appropriate location; and secondly, the mandibular branch of the facial nerve could also be preserved without sacrificing the superficial lobe of the parotid gland. After elevating the platysmal flap, the mandibular branch of the facial nerve was identified and preserved. The tumor was then removed safely en bloc. One-year postoperative follow-up MRI showed no recurrence (Fig. 4).

In conclusion, minimally invasive surgery for certain IMH patients who exhibit small tumors in an appropriate location may be performed safely and efficiently. This type of surgery preserves the cosmetic appearance of the patient, without compromising their prognosis, typically resulting in no tumor recurrence or facial nerve dysfunction.

\section{References}

1. Watson WL and McCarthy WD: Blood and lymph vessel tumors. A report of 1056 cases. Surg Gynecol and Obstet 71: 569-588, 1940.

2. Clemis JD, Briggs DR and Changus GW: Intramuscular hemangioma in the head and neck. Can J Otolaryngol 4: 339-346, 1975.

3. Narayanan CD, Prakash P and Dhanasekaran CK: Intramuscular hemangioma of the masseter muscle: A case report. Cases J 2: 7459, 2009.

4. Odabasi AO, Metin KK, Mutlu C, Başak S and Erpek G: Intramuscular hemangioma of the masseter muscle. Eur Arch Otorhinolaryngol 256: 366-369, 1999.

5. Zengin AZ, Celenk P and Sumer AP: Intramuscular hemangioma presenting with multiple phleboliths: A case report. Oral Surg Oral Med Oral Pathol Oral Radiol 115: e32-e36, 2013

6. Wolf GT, Daniel F, Krause CJ and Kaufman RS: Intramuscular hemangioma of the head and neck. Laryngoscope 95: 210-213, 1985.

7. Smith WP, Prince S and Phelan S: The role of imaging and surgery in the management of vascular tumors of the masseter muscle. J Oral Maxillofac Surg 63: 1746-1752, 2005.

8. Mandel L and Surattanont F: Clinical and imaging diagnoses of intramuscular hemangiomas: The wattle sign and case reports. J Oral Maxillofac Surg 62: 754-758, 2004.

9. Hartzell LD and Buckmiller LM: Current management of infantile hemangiomas and their common associated conditions. Otolaryngol Clin N Am 45: 545-556, 2012.

10. Allen PW and Enzinger FM: Hemangioma of skeletal muscle. An analysis of 89 cases. Cancer 29: 8-22, 1972.

11. Rai P, Setia S, Kalra N and Upreti L: Intramuscular vascular malformation of the masseter muscle presenting with turkey wattle sign. Oral Surg Oral Med Oral Pathol Oral Radiol Endod 102: 618, 2006.

12. Okabe Y, Ishikawa $\mathrm{S}$ and Furukawa M: Intramuscular hemangioma of the masseter muscle: Its characteristic appearance on magnetic resonance imaging. ORL J Otorhinolaryngol Relat Spec 53: 366-369, 1991.

13. Rossiter JL, Hendrix RA, Tom LW and Potsic WP: Intramuscular hemangioma of the head and neck. Otolaryngol Head Neck Surg 108: 18-26, 1993. 